\section{Positive Orientation Across Three Cultures}

\author{
Gian Vittorio Caprara', Guido Alessandri', \\ Gisela Trommsdorff ${ }^{2}$, Tobias Heikamp ${ }^{2}$, \\ Susumu Yamaguchi ${ }^{3}$, and Fumiko Suzuki ${ }^{3}$
}

Journal of Cross-Cultural Psychology 43(I) 77-83

(C) The Author(s) 2012

Reprints and permission:

sagepub.com/journalsPermissions.nav DOI: I0.1 I 77/0022022 I | |422257

jccp.sagepub.com

(SAGE

\begin{abstract}
Positive orientation is a new construct posed at the core of positive evaluations about oneself, life, and the future. Previous findings attested to its associations with health, well-being, and achievement. In the present study, the authors traced standard measures of self-esteem, life satisfaction, and dispositional optimism to a common factor attesting to a similar factorial structure in Japan, Germany, and Italy. Partial invariance at both metric and scalar levels further corroborates generality of positive orientation across these 3 countries.
\end{abstract}

\title{
Keywords
}

positive orientation, self-esteem, life satisfaction, optimism, optimal functioning

An extensive amount of research has focused on individual differences in self-esteem (Rosenberg, 1965), life satisfaction (Diener, Emmons, Larsen, \& Griffin, 1985), and dispositional optimism (Carver \& Scheier, 2002), and documented their respective associations with well-being and success across various domains of functioning in different cultures. However, comparisons at the group level indicated, for instance, that Japanese tend to express a lower level of self-esteem than U.S. Americans (Heine, Takata, \& Lehman, 2000). Despite some differences when comparing cultural groups, testing personality constructs in other cultures can help to determine their generalizability and validity. Since self-esteem, life satisfaction, and optimism are commonly related to each other, the hypothesis was formulated that a basic disposition, characterized by a positive orientation towards self, life, and the future, lies at their core (i.e., positive orientation [POS]; Caprara, Steca, Alessandri, Abela, \& McWhinnie, 2010). From this theoretical perspective, POS is seen as a pervasive mode of appraising, viewing, and construing that significantly affects how individuals predispose themselves to actions and experiences. It has been reasoned that humans need a certain amount of POS to cope with the inevitable adversities, failures, and losses of life (Alessandri, Caprara, \& Tisak, in press; Caprara, Steca et al., 2010). Likewise, individual differences in POS may exert important biological and social functions in balancing approach and avoidance tendencies to risk and innovation (Caprara et al., 2009). In a recent study on a sample

\footnotetext{
l“Sapienza," University of Rome, Italy

${ }^{2}$ University of Konstanz, Germany

${ }^{3}$ Department of Social Psychology, University of Tokyo, Japan

\section{Corresponding Author:}

Gian Vittorio Caprara, Department of Psychology, Sapienza, University of Rome, Italy

Email: gianvittorio.caprara@uniromal.it
} 
of Italians, mono- and dizygotic twin pairs answered self-report measures assessing self-esteem, life satisfaction, and optimism. Multivariate genetic analyses based on structural equation modeling revealed high correlations of genetic factors (i.e., amount of variance two traits share due to the same genetic factors) between .80 and .87 for self-esteem, life satisfaction, and optimism, suggesting that a common genetic factor affects the three dimensions simultaneously (Caprara et al., 2009). Measurement findings attested to a conceptual model positing a common latent dimension at the core of standard measures of self esteem, life satisfaction, and dispositional optimism. Cross-sectional findings converge with longitudinal findings in pointing to POS as a basic predisposition that accounts for individuals' adjustment and achievement to a considerable extent. In reality little variance is left to self-esteem, life satisfaction, and optimism once the amount of variance due to POS is kept under control as a study with participants from Italy and Canada revealed (Caprara, Alessandri, \& Barbaranelli, 2010).

In this article, using data from three different countries (i.e., Italy, Japan, Germany), we aim to further corroborate our conceptual model across cultures, tracing self-esteem, life satisfaction, and dispositional optimism to a common latent dimension identified with POS across the three countries. The generality of our model will provide further empirical evidence to support arguments pro-POS as a basic personality disposition. In reality, the three countries differ widely in cultural features like language, religion, socialization practices, traditions, and habits (Hofstede, 2001; Schwartz, 2007). In particular, a vast debate is still ongoing on the extent to which Japanese culture differs from Western cultures in the way people evaluate themselves and their life (Heine et al., 2007). Japanese people have reported lower self-esteem in comparison to Americans when using explicit measures (i.e., questionnaire based), but no differences have been found when implicit measures (i.e., reaction time based) have been taken into account (cf., Yamaguchi et al., 2007). Regarding life satisfaction, Japan ranked 34th despite being the third strongest economic power in the world. Although wealth does not necessarily carry happiness, it is surprising that Japanese who outperform people from other countries in school and economic achievement do not represent personality characteristics that are commonly associated with success (Kusago, 2007).

We reasoned that cross-cultural generality of basic personality dispositions should be demonstrated by a high degree of factorial structure invariance across cultures. In accordance with this reasoning, we expected to replicate the same measurement model across cultures. In line with previous studies, we assumed, furthermore, that Japanese would score lower than Italians and Germans in self-esteem, life satisfaction, optimism, and POS, because cultural differences between these groups may impinge on styles of self-representations (e.g., Yamaguchi et al., 2007). However, we expected no substantial differences between Italian and Germans.

\section{Method}

\section{Participants}

Italian sample. The Italian participants were 243 men and 321 women from Genzano, a residential community near Rome. They ranged in age from 19 to 29 years $(M=21.68, S D=1.60)$. Participants were students coming from families involved in an ongoing longitudinal study in that community. The occupational socioeconomic distribution of the families of origin matched the national profile. Many participants were university students (in general from Sapienza, the university of Rome), and almost all lived with their parents and were of Italian origin.

Japanese sample. The Japanese participants were college students (98 men, 105 women) ranging in age from 18 to 28 years $(M=19.21, S D=1.40)$. They were from three universities: University of Tokyo $(n=62)$, Sapporo University $(n=34)$, and Nihon Fukushi University $(n=107)$. 
German sample. Participants were 185 male and 298 female undergraduate students at the University of Konstanz. The students were between 18 and 26 years of age $(M=21.43, S D=1.45)$.

\section{Procedure}

In Italy, participants were invited to participate in the study by phone and three female researchers collected all questionnaires during specially scheduled sessions in a school. In Japan, participants were asked to participate in the study at psychology classes on a voluntary basis. The participants completed the questionnaire in the classroom. In Germany, participants were recruited from introductory courses in their respective fields of study. They either completed the questionnaires after the lecture or filled in the questionnaires at home and sent them back by mail (13.9\%). For compensation, participants could take part in a lottery of vouchers worth 20 Euros. Psychology students alternatively received course credit.

\section{Instruments}

Self-esteem. Self-esteem was assessed by the 10 items of the Rosenberg's General Self-Esteem Scale (RGSES; Rosenberg, 1965), using a 4-point Likert-type scale (1 = strongly disagree, $4=$ strongly agree).

Life satisfaction. Participants rated life satisfaction on a 7-point Likert-type scale ( $1=$ strongly disagree, 7 = strongly agree) by using the five items of the Satisfaction With Life Scale (SWLS; Diener et al., 1985).

Optimism. Optimism was measured by using the 10-item set from the Life Orientation Test (LOT-R; Scheier, Carver, \& Bridges, 1994). In particular, the six scale items (4 items were filler) measured participants' expectations about their future and their general sense of optimism. In each country, previously validated and well-established translations of the respective instruments were used to assess self-esteem, life satisfaction, and optimism. Cronbach's alphas were, respectively, $.88, .88$, and .81 for the Italian version; $.90, .80$, and .78 , for the German version; and $.84, .78$, and .74 for the Japanese version of these instruments.

\section{Statistical Analysis}

First, we examined the factor structure of the measures of the POS model separately in each group using linear confirmatory factor analysis (CFA) in LISREL 8.71 (Jöreskog \& Sörbom, 2004). The following criteria were employed to evaluate the goodness of fit: chi-square likelihood ratio statistic, non-normed fit index (NNFI), comparative fit index (CFI), the root mean square error of approximation (RMSEA) with associated confidence intervals, and the standardized root mean square residual (SRMR). The chi-square test is sensitive to sample size. Obtaining a nonsignificant chi-square becomes increasingly unlikely with complex models and large sample sizes, even for trivial group differences or model misspecifications. We chose cutoff values of .95 for CFI, .06 for RMSEA, and.08 for the SRMR. After the fit of the POS model was established separately for each of the three groups, we used multigroup CFAs (MCFA) to examine measurement invariance (Meredith \& Teresi, 2006). To test differences among the base model and the more restricted models, we calculated restricted chi-square tests $\left(\Delta \chi^{2}\right)$ with an $\alpha$ level of .01. If a step resulted in a significant chi-square difference test, the particular restriction was rejected and parameters with the highest modification index (MI; Jöreskog \& Sörbom, 2004) were sequentially estimated. When partial measurement invariance was established (Meredith \& Teresi, 2006), the sequence was continued. 


\section{Results}

\section{Fit of the Conceptual Model}

Data analyses revealed a close fit of the hypothesized model within each of the three samples: Japan: $\chi^{2}(170)=266.70, p<.01, \mathrm{NNFI}=.967, \mathrm{CFI}=.973, \mathrm{RMSEA}=.054(\mathrm{CI}=.041-.066)$, SRMR $=.058$; Germany: $\chi^{2}(170)=326.06, p<.01, \mathrm{NNFI}=.987, \mathrm{CFI}=.989, \mathrm{RMSEA}=.044$ $(\mathrm{CI}=.037-.051), \mathrm{SRMR}=.044$; Italy: $\chi^{2}(170)=266.70, p<.01, \mathrm{NNFI}=.978, \mathrm{CFI}=.982$, $\mathrm{RMSEA}=.046(\mathrm{CI}=.050-.065), \mathrm{SRMR}=.058$. Although the chi-square was large and significant, alternative fit indexes satisfied the more restrictive cutoff criteria. In all countries, item loadings on self-esteem, life satisfaction, and optimism were significant and above.33 (except for some loadings on the three method effect factors). Moreover, the loadings of the three first order factors on POS were large and significant (all above .69).

\section{Configural Invariance}

Having established the good fit of the hypothesized model, we proceeded with examining configural invariance and estimated this model in all three groups simultaneously. The configural model showed a good fit to the data: $\chi^{2}(510)=1,009.30, p<.01$, NNFI $=.983, \mathrm{CFI}=.986$, $\mathrm{RMSEA}=.048(\mathrm{CI}=.044-.053), \mathrm{SRMR}=.061$ (Figure 1).

\section{Metric Invariance}

When we tested metric invariance, constraining the first order factor loadings to be equal across the three groups, the change in overall chi-square was significant, $\Delta \chi^{2}(61)=258.76, p<.01$. Two loadings on the self-esteem factor were relaxed to be different from the Italian sample (i.e., RSGP3 and RSGN2). Moreover, a loading from the negative method factor was relaxed to be different across the three countries (i.e., RSEP1), and an additional item from the negative method factor was relaxed to be different from the Italian sample (i.e., RSEN4). Then, the chisquare difference tests supported the viability of the partial metric invariance hypothesis, $\Delta \chi^{2}(54)=64.97, p=.15$. When second order loadings were constrained to be equal across countries, the chi-square difference test became nonsignificant, $\Delta \chi^{2}(4)=5.37, p=.25$. Thus, the hypothesis of equal second order structure coefficients was not rejected.

\section{Scalar Invariance}

We then tested first order scalar invariance by constraining the first order intercepts as well. The chi-square difference test between this model and the less constrained model was significant, $\Delta \chi^{2}(41)=253.46, p<.01$, suggesting that full strong invariance did not hold at the first order level. Intercepts for two items from the LOT-R (i.e., LOTP3 and LOTP4) and one from the RGSES (i.e., RSGN1) were relaxed to be different across countries. Moreover, one intercept from the LOT-R (i.e., LOTN2) and two intercepts from the RGSES (i.e., RSGP4 and RSGN5) were relaxed to be different from the Italian sample. Then, the chi-square difference test was no longer significant, $\Delta \chi^{2}(27)=38.71, p=.07$. Finally, we constrained the second order intercepts to be equal across countries (second order scalar invariance). Again, the chi-square difference test between this model and the less constrained model was significant, $\Delta \chi^{2}(4)=247.07, p<.01$. Only the intercept of self-esteem on the Japanese sample needed to be relaxed to obtain a less restrictive model with a chi-square value not significantly different from the previous model, $\Delta \chi^{2}(3)=6.83, p=.08$. The above results support second order partial scalar invariance: accordingly, means can be meaningfully compared. 


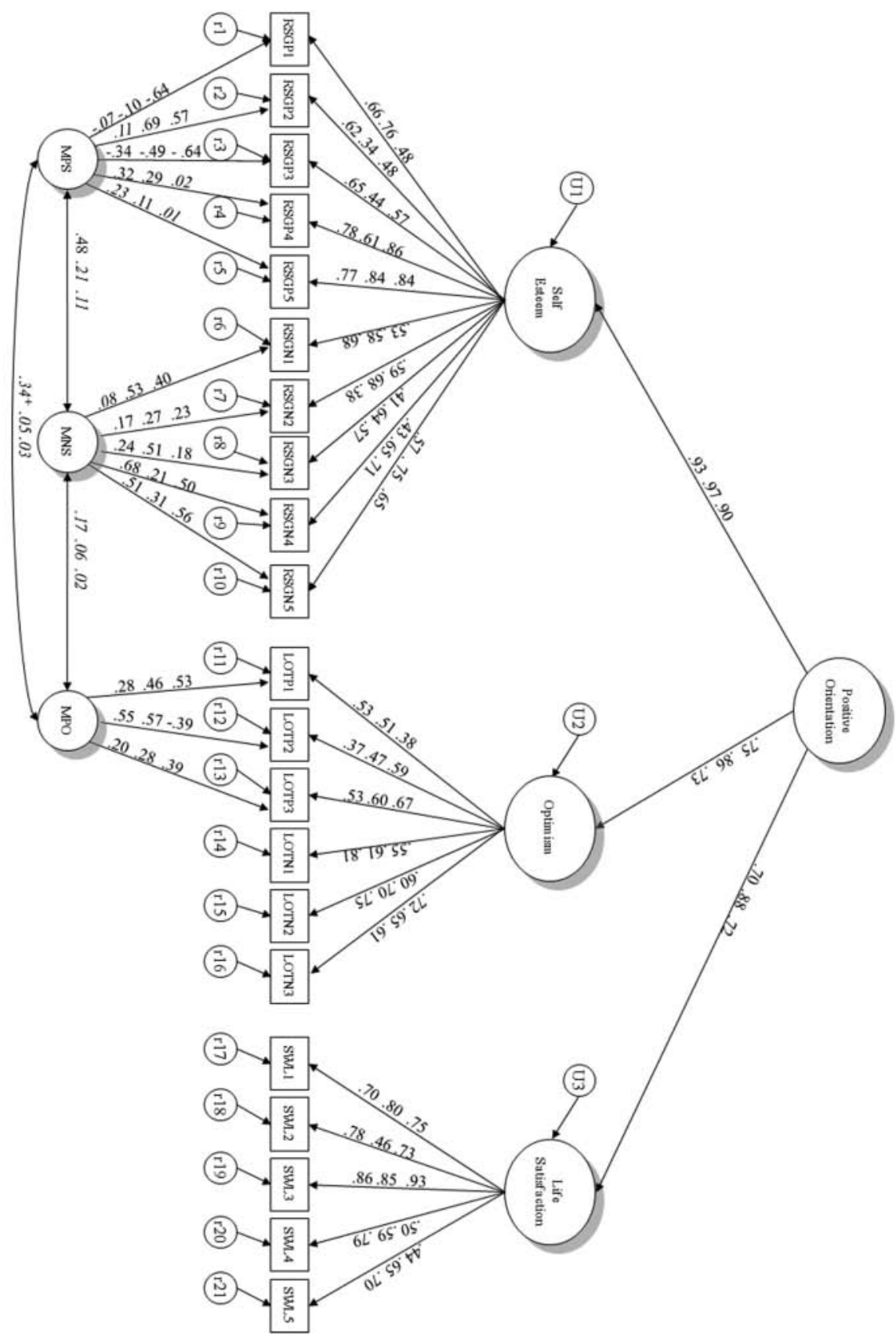

Figure I. The Measurement Model of Positive Orientation With Standardized Parameter Estimates Resulting From Configural Invariance

Note. The first coefficient (starting from the left) is for Japan, the second is for Germany, and the third is for Italy. Nonsignificant paths are in italics. $\mathrm{r} \mid-\mathrm{r} 2 \mathrm{I}=$ unique items and error variance; $\mathrm{U}=$ factor unique variance; $M P S=$ method factor associated with positively worded items from the RSGE; MNS = method factor associated with negatively worded items from the RSGE; MPO = method factor associated with positively worded items from the LOT-r. ${ }^{+} p<.10$. 


\section{Mean Level Comparisons}

Differences in mean levels were further investigated with ANOVAs and Tukey post hoc test, conducted on the individual's factor scores obtained by previous MCFA.

Self-esteem. Japanese $(M=-1.19, S D=.82)$ scored significantly lower, $F(1,1,247)=241, p<.01$, $\eta^{2}=.28$, than Italians $(M=.31, S D=.83 ; d=-2.09)$ and Germans $(M=.14, S D=.88 ; d=-2.99)$. Moreover, Germans scored significantly lower than Italians $(d=-.36)$.

Life satisfaction. Japanese $(M=-1.06, S D=.93)$ scored significantly lower, $F(1,1,247)=177.54$, $\left.p<.01, \eta^{2}=.22\right)$ than Italians $(M=.28, S D=.28 ; d=-1.68)$ and Germans $(M=.12, S D=.80 ; d=$ -2.33). Moreover, Germans scored significantly lower than Italians $(d=-.30)$.

Optimism. Japanese $(M=-.49, S D=.99)$ scored significantly lower, $F(1,1,247)=30.98, p<.01$, $\eta^{2}=.05$, than Italians $(M=.10, S D=.99 ; d=-.69)$ and Germans $(M=.09, S D=.99 ; d=-1.07)$.

Positive orientation. Japanese $(M=-1.07, S D=.85)$ scored significantly lower, $F(1,1,247)=$ $179.30, p<.01, \eta^{2}=.23$, than Italians $(M=.27, S D=.88 ; d=-1.76)$ and Germans $(M=.13$, $S D=.89, d=-2.59)$. Moreover, Germans scored significantly lower than Italians $(d=-.27)$.

\section{Discussion}

The present study offers a further contribution to a theory of POS as a pervasive psychological mode of facing reality, framing events, and processing personal and interpersonal experiences over time and across life circumstances. Results corroborate a measurement model tracing traditional measures of self-esteem, life satisfaction, and optimism to a common latent dimensions while showing a common pattern of relations across three cultures (i.e., Italy, Germany, Japan) that widely differ in their narratives about self, life, and time perspective (e.g., Hofstede, 2001; Schwartz, 2007). Likely individual differences in people's evaluations of own self, life, and future rest upon a common basic personality disposition to hold a positive view of oneself in a world to color experiences, frame the events, and predispose to action accordingly. This view is needed to cope with setbacks and losses and to preserve interest in life, despite the unavoidability of death. As self-esteem proved to be the major component of POS across cultures, present findings corroborate previous findings assigning to self-esteem a pivotal role in coloring the way people view the world and in driving both optimism and life satisfaction (Alessandri et al., in press; Caprara, Alessandri et al., 2010). Findings corroborate our expectations showing a similar pattern of relations among self-esteem, life satisfaction, and optimism across countries even while significant differences in means between Eastern and Western countries have occurred. Japanese scored lower than Italians and Germans in positive orientation as in self-esteem, life satisfaction, and dispositional optimism. These findings are in line with previous findings revealing that Japanese tend to report lower self-esteem in comparison with North Americans (Yamaguchi et al., 2007), despite showing a similar pattern of associations between self-esteem and psychological well-being. Also, these findings are consistent with a lower life satisfaction reported by Japanese in comparison with people of other countries who show conditions of life much less satisfactory (Kusago, 2007). In this respect, a study by Yamaguchi et al. (2007) is particularly informative about the tendency of Japanese to take a low profile when interacting with others while paying a large tribute of modesty to others' respect and concern.

\section{Conclusion}

As previous findings attest to POS as a major determinant of well-functioning (Caprara, Steca et al., 2010), additional research is needed to further clarify its unique contribution to people's subjective well-being across cultures. Moreover, systematic replication with samples from different cultures using different sets of measures of self-esteem, life satisfaction, and optimism (e.g., using implicit and explicit measures) is necessary to further corroborate the universality of POS. 
Likewise further research is needed to clarify the extent to which POS may be a target for interventions aimed to promote human strengths and whether similar strategies could enhance POS and its beneficial effects across cultures (cf., Caprara, Alessandri, \& Barbaranelli, 2010).

\section{Acknowledgments}

This study was supported in part by grants from the Italian Italian Ministero dell'Istruzione, dell'Università della Ricerca (MIUR) 2002 and 2005, and from Sapienza University of Rome to Gian Vittorio Caprara. This research was also supported by a grant from the German Research Foundation (DFG GZ, TR 169/142) to Gisela Trommsdorff as part of the project Developmental Conditions of Intentionality and its Limits within the interdisciplinary research group Limits of Intentionality at the University of Konstanz, Germany. We thank Catherine Gunzenhauser for her help in data collection and preliminary data analyses. We are grateful to Holly Bunje for editing the manuscript.

\section{Declaration of Conflicting Interests}

The author(s) declared no potential conflicts of interest with respect to the research, authorship, and/or publication of this article.

\section{Funding}

The author(s) received no financial support for the research, authorship, and/or publication of this article.

\section{References}

Alessandri, G., Caprara, G. V., \& Tisak, J. (in press). Further explorations on the unique contribution of Positive Orientation to optimal functioning. European Psychologist.

Caprara, G. V., Alessandri, G., \& Barbaranelli, C. (2010). Optimal functioning: Contribution of self-efficacy beliefs to positive orientation. Psychotherapy and Psychosomatics, 79, 328-330.

Caprara, G. V., Fagnani, C., Alessandri, G., Steca, P., Gigantesco, A., Cavalli Sforza, L. L., et al. (2009). Human optimal functioning: The genetics of positive orientation towards self, life, and the future. Behaviour Genetics, 39, 277-284.

Caprara, G. V., Steca, P., Alessandri, G., Abela, J. R. Z., \& McWhinnie, C. M. (2010). Positive orientation. Epidemiologia e Psichiatria Sociale, 39, 63-71.

Carver, C. S. \& Scheier, M. F. (2002). Optimism. In C. R. Snyder \& J. L. Lopez (Eds.), Handbook of positive psychology (pp. 231-243). New York: Oxford University Press.

Diener, E., Emmons, R. A., Larsen, R. J., \& Griffin, S. (1985). The satisfaction with life scale. Journal of Personality Assessment, 49, 71-75.

Heine, S. J., Takata, T., \& Lehman, D. R. (2000). Beyond self-presentation: Evidence for self-criticism among Japanese. Personality and Social Psychology Bulletin, 26, 71-78.

Hofstede, G. (2001). Culture's consequences: Comparing values, behaviors, institutions and organizations across nations (2nd ed.). Thousand Oaks, CA: SAGE.

Jöreskog, K., \& Sörbom, D. (2004). LISREL 8.71 [Computer software]. Chicago: SSI.

Kusago, T. (2007). Rethinking of economic growth and life satisfaction in Post-WWII Japan: A fresh approach. Social Indicators Research, 81, 79-102.

Meredith, W., \& Teresi, J. A. (2006). An essay on measurement and factorial invariance. Medical Care, 44, 69-77.

Rosenberg, M. (1965). Society and the adolescent self-image. Princeton, NJ: Princeton University Press.

Scheier, M. F., Carver, C. S., \& Bridges, M. W. (1994). Distinguishing optimism from neuroticism. Journal of Personality and Social Psychology, 67, 1063-1078.

Schwartz, S. H. (2007). Value orientations: Measurement, antecedents and consequences across nations. In R. Jowell, C. Roberts, R. Fitzgerald, \& G. Eva (Eds.), Measuring attitudes cross-nationally: Lessons from the European Social Survey (pp. 169-203). London: SAGE.

Yamaguchi, S., Greenwald, A. G., Banaji, M. R., Murakami, F., Chen, D., et al. (2007). Apparent universality of positive implicit self-esteem. Psychological Science, 18, 498-500. 